\title{
Research on Hydrophobic Properties of Grating Structure on Monocrystalline Silicon Fabricated Using Micromachining
}

\author{
Wenyu Ding, ${ }^{1,2}$ Ziyang Cao $\mathbb{D}^{1,2}$ Bangfu Wang, ${ }^{1,2}$ Shunjie $\mathrm{Xu}^{1,2}$ and Zhongwang Wang ${ }^{1,2}$ \\ ${ }^{1}$ College of Mechanical Engineering, Suzhou University of Science and Technology, Suzhou, Jiangsu 215009, China \\ ${ }^{2}$ Suzhou Key Laboratory of Precision and Efficient Machining Technology, Suzhou, Jiangsu 215009, China \\ Correspondence should be addressed to Ziyang Cao; dukeczy@nuaa.edu.cn
}

Received 17 February 2019; Revised 10 April 2019; Accepted 18 April 2019; Published 2 May 2019

Academic Editor: Antonio Riveiro

Copyright (c) 2019 Wenyu Ding et al. This is an open access article distributed under the Creative Commons Attribution License, which permits unrestricted use, distribution, and reproduction in any medium, provided the original work is properly cited.

In this paper, micromilling was used to process the surface of single crystal silicon, and six different structural parameters of the grating structure were tested to get the contact angle in a different wettability. The contact angle obtained by the experiment was compared with the theoretical values of the Wenzel and Cassie model optimized based on the characteristics of micromilling. The relationship between structural parameters and wettability was verified. First, the contact angle of the parallel grating structure was greater than the vertical direction, which was influenced by the solid-liquid interface tension. Second, the hydrophobicity of the specimen was in good agreement with the predicted trend of the optimized prediction model $\mathrm{C}$ when the width of the convex is reduced. The support of the theoretical model to the experimental results is instructive to the construction of the structure. In addition, the molecular dynamics were used to verify the hydrophobicity of grating structures from a molecular point of view.

\section{Introduction}

The wetting property of any solid material's surface refers to the spreading ability of a given liquid on the solid surface, which is characterized by the contact angle. The contact angle at the solid-liquid interface is a result of the balance between the cohesion and adhesion forces acting at the solid-liquid-gas three-phase contact line [1-3]. It is also an important technical index of hydrophobic performance. In general, the surface is called hydrophobic when it possesses water contact angle (WCA) equal or higher than $90^{\circ}$, while it is considered hydrophilic if the WCA is below $90^{\circ}$. There are two cases of extreme wetting and nonwetting, where the WCA can approach $0^{\circ}$ or $180^{\circ}$, respectively, called superhydrophilicity and superhydrophobicity [4-7]. The hydrophobic surface of monocrystalline silicon has great application value in solar energy for its heat transfer performance, which brings great convenience to people's production and life. In addition, hydrophobic surfaces have important application value in many industrial fields, including self-cleaning surfaces, anti-icing/ frosting systems, and anti-biofouling coatings, which are of great value to the development of society [8-10]. The multilevel structure and low surface energy materials, composed of micro- and nanoscale structures, can make the surface of any material hydrophobic or superhydrophobic [11-13].

Some materials, such as quartz glass, monocrystalline silicon, and diamond, are widely used in high-tech fields such as civil and national defense for their high hardness, high strength, and stable chemical properties in the context of normal temperature. Among them, the monocrystalline silicon has the unique advantages of high thermal conductivity, low density, low expansion coefficient, and excellent optical performance. It also plays an important role in solar energy technology as the monocrystalline silicon is the main material for the production of solar cells [14].

At present, chemical and physical treatment methods account for the majority of the various methods for preparing hydrophobic surfaces. Razavi et al. [15] used oxidation to make microstructures on copper. Wang et al. [16] used ion etching to make microstructures for Si. However, neither of them can control the structural morphology. Chen et al. [17] developed a rapid one-step electrodepositing process to fabricate the superhydrophobic cathodic surface on the copper plate. Liu et al. [18] used the electrodeposition process 
to fabricate the hydrophobic surface on Cu. Yang et al. [19] and Gurav et al. [20] developed some new coatings using a sol-gel method. Zorba et al. [21, 22] used femtosecond laser to tailor the wetting response of silicon surfaces. However, the parameters of those physically and chemically patterned structures are not controllable, and the ambient environments of the experiment are very specific [23]. As a result, it is difficult to study the rules of variation between surface structure and wettability accurately. The processing is very expensive, which means the large-scale production is difficult [24]. At the same time, the size of the milling tool is reduced gradually with the study of the micromachining mechanism. It is possible to study the wettability of micron-sized microstructures by micromilling [25]. The controllability and repeatability of the machining process is good. Besides, the high-speed micromilling technology is widely used in machinery manufacturing industry. This technology is known for high processing precision, high processing efficiency, simple preparation process, and relatively low preparation cost and arbitrary shape of three-dimensional structure processing [26]. In this context, the structure parameters and morphology of any surface can be determined accurately with micromilling technology. This processing method ensures to study the rules of variation between surface structure and wettability accurately. The processing is also simplified. Moreover, the theoretical models cannot be applied to all structures, which resulted in a large deviation between theoretical and experimental values of contact angle [27].

In this paper, the structure of the monocrystalline silicon surface was changed and the appropriate grating structure was selected to obtain hydrophobicity, which improves the thermal conductivity and solar energy efficiency and enhances the anticorrosion ability [28]. First, a theoretical model suitable for micromilling characteristics was established to study the effect of micron particle size on the contact angle of droplets resting on the silicon surfaces. Second, the micrograting structure was fabricated by high-speed micromilling $[29,30]$. Third, the contact angle measured after machining was compared with the analysis under molecular dynamics simulation, and the influence of microstructure size parameters on the contact angle was studied.

\section{Construction of the Prediction Model}

2.1. Basic Principle of Hydrophobicity. The wetting phenomenon reflects the equilibrium relation of three-phase interfacial tension between solid, liquid, and gas. In 1805, the functional correspondence between the static contact angle of a smooth surface and the interfacial tension is derived for the first time by Young [31], which is based on the thermodynamic equilibrium theory. And this functional relationship is named "Young's equation." Young's equation is proposed under the condition of neglecting the line tension and the gravity of water droplets. It is used to measure the ideal contact angle of the absolutely smooth surface. Contact angle $\theta$ with a smooth surface is calculated as follows [32]:

$$
\cos \theta=\frac{\sigma_{\mathrm{sv}}-\sigma_{\mathrm{sl}}}{\sigma_{\mathrm{lv}}}
$$

where $\sigma_{\mathrm{sv}}, \sigma_{\mathrm{sl}}$, and $\sigma_{\mathrm{lv}}$ are the interfacial tensions of the solidgas phase, solid-liquid phase, and gas-liquid phase, respectively, which are shown in Figure 1.

Actually, the surface of natural solids has a certain degree of roughness. The Wenzel [33] model and the Cassie-Baxter [34] model are both proposed to estimate the effect of surface roughness. The Wenzel model shows the case that the droplet maintains contact with the entirety of the rough surface, which results in the increase of the interfacial contact area. In contrast, the Cassie model indicates the case that the droplet skips between the peaks of the roughness and leaves the interspace of solid-liquid and liquid-gas interfaces below it. The structures are shown in Figure 2.

The contact angle $\theta_{\mathrm{w}}$ of the Wenzel model is calculated as follows:

$$
\cos \theta_{\mathrm{w}}=r \cos \frac{\sigma_{\mathrm{sv}}-\sigma_{\mathrm{sl}}}{\sigma_{\mathrm{lv}}} .
$$

The contact angle $\theta_{c}$ of the Cassie-Baxter model is calculated as follows:

$$
\cos \theta_{\mathrm{c}}=f+\cos \frac{\sigma_{\mathrm{sv}}-\sigma_{\mathrm{sl}}}{\sigma_{\mathrm{lv}}} f-1
$$

In formulas (2) and (3), $r$ and $f$ are the ratio of the actual solid-liquid contact area to the horizontal projection area of the droplet, which was named after roughness factors [35]. The Cassie model plays an important role in enhancing the hydrophobic properties of the microstructure surface. As a result, the Cassie model is an ideal model to describe the superhydrophobicity of the solid surfaces towards the liquid (water) [36].

\subsection{Construction of Prediction Model Based on Parameters.} The schematic diagram of the grating structure is shown in Figure 3, where $a$ is the convex platform width of the grating structure, $b$ represents the spacing width of the grating structure, $h$ is the height of convex, and $l$ represents the length of the contact surface of the droplet on the surface.

The precise prediction model suitable for micromilling characteristics was established on the basis of Gibbs free energy. The surface area of the convex platform under droplets is $S=\left(\pi l^{2} /(2 \rho)^{2}\right) \cdot(2 l / a) \cdot(2 l /(a+b)) \cdot a^{2}=\pi a l^{2} /$ $(a+b)$. Gibbs free energy can be associated with the contact angle due to the interfacial free energy, and surface tension is equal in value. In this paper, the prediction models are named prediction model $\mathrm{W}$ and prediction model $\mathrm{C}$, respectively.

The contact areas of solid-liquid, gas-liquid, and solidgas interfaces of prediction model $\mathrm{W}$ are as follows:

$$
\begin{aligned}
& A_{\mathrm{sl}}=\pi l^{2}+\frac{S}{a^{2}} \cdot 2 a h=\pi l^{2}+\frac{2 \pi h l^{2}}{a+b}, \\
& A_{\mathrm{lv}}=\int_{0}^{\theta_{\mathrm{w}^{\prime}}} 2 \pi l R d \theta=\frac{2 \pi l^{2}}{1+\cos \theta_{\mathrm{w}^{\prime}}}, \\
& A_{\mathrm{sv}}=0 .
\end{aligned}
$$




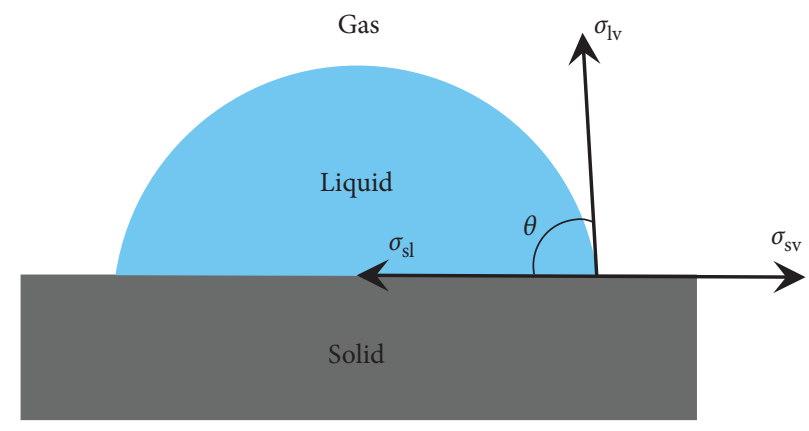

Figure 1: Schematic diagram of droplet contact angle on a smooth solid surface.

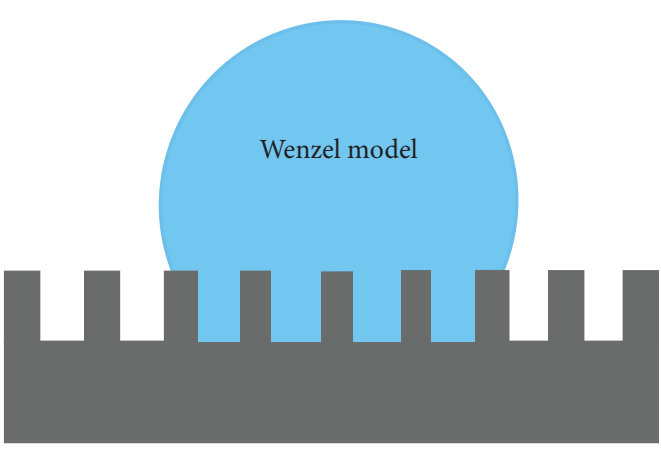

(a)

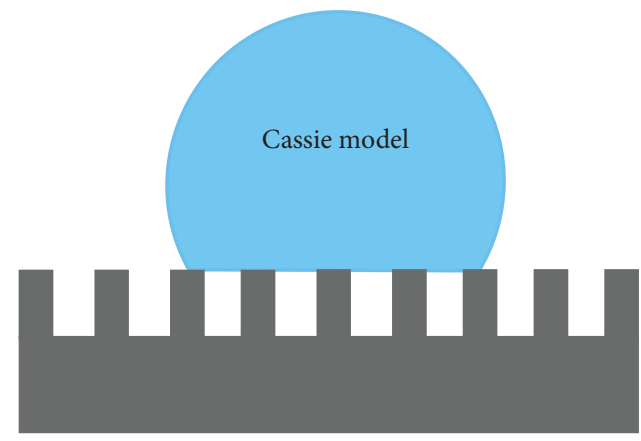

(b)

Figure 2: Droplet diagram of Wenzel and Cassie models on hydrophobic surfaces: (a) Wenzel model; (b) Cassie-Baxter model.

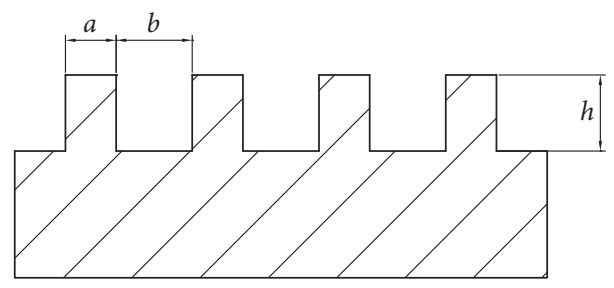

(a)

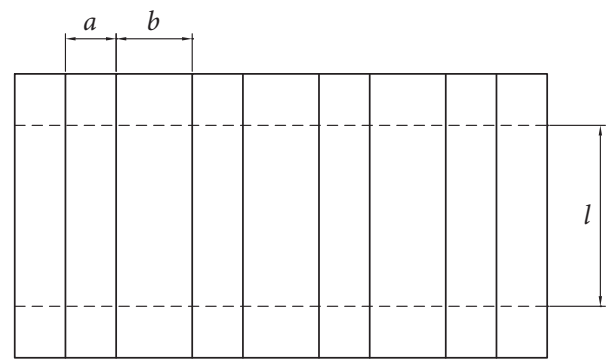

(b)

Figure 3: The diagram of the structural parameter of the grating structure.

According to equation (1), the relationship between surface tension and contact areas can be expressed as follows:

$$
\cos \theta=\frac{\sigma_{\mathrm{sv}}-\sigma_{\mathrm{sl}}}{\sigma_{\mathrm{lv}}}=\frac{d A_{\mathrm{lv}}}{d A_{\mathrm{sl}}}=\frac{(a+b) \cos \theta_{\mathrm{w}^{\prime}}}{a+b+2 h} .
$$

Therefore, the relationship between contact angle and geometric parameters in prediction model $\mathrm{W}$ can be shown as follows:

$$
\cos \theta_{\mathrm{w}^{\prime}}=\left(1+\frac{2 h}{a+b}\right) \cos \theta=r \cos \theta .
$$


In contrast, the contact areas of prediction model $\mathrm{C}$ are different from prediction model $\mathrm{W}$ :

$$
\begin{aligned}
A_{\mathrm{sl}}= & S=\frac{\pi a l^{2}}{a+b}, \\
A_{\mathrm{lv}}= & \int_{0}^{\theta_{c^{\prime}}} 2 \pi l R d \theta+\left(\pi l^{2}-S_{\mathrm{sl}}\right)=\frac{2 \pi l^{2}}{1+\cos \theta_{\mathrm{c}^{\prime}}} \\
& +\pi l^{2}-\frac{\pi a l^{2}}{a+b}, \\
A_{\mathrm{sv}}= & A_{\text {total }}-A_{\mathrm{sl}} .
\end{aligned}
$$

Thus, the relationship between surface tension and the contact areas of prediction model $\mathrm{C}$ can be represented as follows:

$$
\cos \theta=\frac{\sigma_{\mathrm{sv}}-\sigma_{\mathrm{sl}}}{\sigma_{\mathrm{lv}}}=\frac{d A_{\mathrm{lv}}}{d A_{\mathrm{sl}}}=\frac{(a+b) \cos \theta_{\mathrm{c}^{\prime}}}{a}+\frac{a+b}{a}-1 .
$$

According to equation (8), the relationship between the contact angle and geometric parameters can be obtained as follows:

$$
\cos \theta_{\mathrm{c}^{\prime}}=\frac{a}{a+b} \cos \theta+\frac{a}{a+b}-1=f+\cos \theta f-1 .
$$

Figure 4 shows the relationship between structural parameters and contact angle, which were derived from equations (6) and (9).

It can be seen that when the height and spacing width of the convex are constant, the contact angle of prediction model $\mathrm{W}$ increases with the increase of convex platform width, while the contact angle of prediction model $\mathrm{C}$ decreases. Moreover, when the height and platform width of convex are fixed, the contact angle of prediction model $\mathrm{W}$ goes up and so does of prediction model C.

\section{Experimental Method}

The experimental material was selected to be polished monocrystalline silicon with 100 crystal surface. The diameter of it was $25 \mathrm{~mm}$, and the thickness was $400 \mu \mathrm{m}$ [37]. However, the silicon wafer was too thin to fix it on the worktable by the traditional clamping method. As a result, a copper with the size of $25 \mathrm{~mm} * 25 \mathrm{~mm} * 25 \mathrm{~mm}$ was fixed on the vise. Then, the upper surface of the copper was smoothed with a $3 \mathrm{~mm}$ diameter milling cutter. At last, the silicon wafer was pasted on the copper smoothly and tightly. The workpieces were machined on high-speed milling center, as shown in Figure 5, with a double-edged micromilling cutter. The milling cutter was a diameter of $0.2 \mathrm{~mm}$ with diamond coating. The experiments were carried out under the condition that the spindle speed was $48000 \mathrm{r} / \mathrm{min}$ and constant feed speed was $6 \mathrm{~mm} / \mathrm{min}$ [38]. 15 grating grooves of different sizes were processed on the intermediate surface of the workpieces to form the grating structure.

After processing, these samples were cleaned in an ultrasonic cleaner containing ethanol for $5 \mathrm{~min}$ to remove the burr and impurities, followed by a $10 \% \mathrm{HF}$ aqueous treatment to remove the oxide grown on the surface of these samples. Then, the samples were air-dried naturally to reduce the external errors for the measurement of contact angle. All the apparent morphology of samples was observed with VHX-5000 Superhigh magnification lens zoom 3D microscope (VHX-5000, Keyence, Japan). The contact angle of the grating structure was measured by an optical contact angle measuring instrument (OCA, DataPhysics, Germany). The middle of the grating structure was selected as the measuring point of the contact angle to ensure that the experimental data have the same measuring point. The contact angle of each specimen was measured five times to ensure the reliability of these data [39].

\section{Result and Analysis}

During processing, both the spacing width $b$ and the height of convex $h$ were fixed. The geometrical parameters of the design of the grating structure are shown in Table 1. Figure 6 shows the obtained grating structure surface for $\mathrm{Si}$.

Figure 7 shows the obtained contact angles for the sample. The contact angle of the unprocessed monocrystalline silicon is $56.3^{\circ}$. Besides, the theoretical contact angle is calculated based on equations (6) and (9). All of the data are shown in Table 2.

According to Table 2, the variation curve of each contact angle is shown in Figure 8. Comparing Table 2 and Figure 8, it can be seen that the contact angle of the six samples is greatly improved than the original specimen.

From Figure 8, it can be seen that a huge mismatch in the value of the contact angle between the vertical direction and the parallel direction. In these six specimens, the contact angle in the parallel direction is always greater than the vertical direction, and the average difference is $51.85^{\circ}$. It is clear that the wetting phenomenon is related to the three-phase equilibrium of the solid, liquid, and gas. In this paper, there is no convex platform constraining the droplets on both sides [40]. The solid-liquid interfacial tension is lower in the direction vertical to the groove than the parallel direction. The droplet will tend to spread completely on the solid in the vertical direction as $\left(\sigma_{\mathrm{sv}}-\sigma_{\mathrm{sl}}\right)$ is larger than $\sigma_{\mathrm{lv}}$. As a result, the hydrophobic anisotropy will occur in the grating structure. The sphere is easier to form in the parallel direction, which will cause an increase in the contact angle.

The trend of the contact angle in the parallel direction was in good agreement with the value of prediction model C. Figure 7(b) shows that there is a gap between the droplet and the structure of surface, which means this structure matches prediction model C [41]. The established model predicts the contact angle accurately. In prediction model $\mathrm{C}$, the contact angle decreased with the increase of the convex platform width and so does the contact angle of the parallel direction.

It can be seen from Figure 8 that the contact angle in two directions increases as the width of the convex changes from $20 \mu \mathrm{m}$ to $60 \mu \mathrm{m}$. Among these specimens, the hydrophobic property of sample 1 was the best. Therefore, the smaller the convex platform width, the larger the contact angle, and the hydrophobic performance of the structure was stronger. 


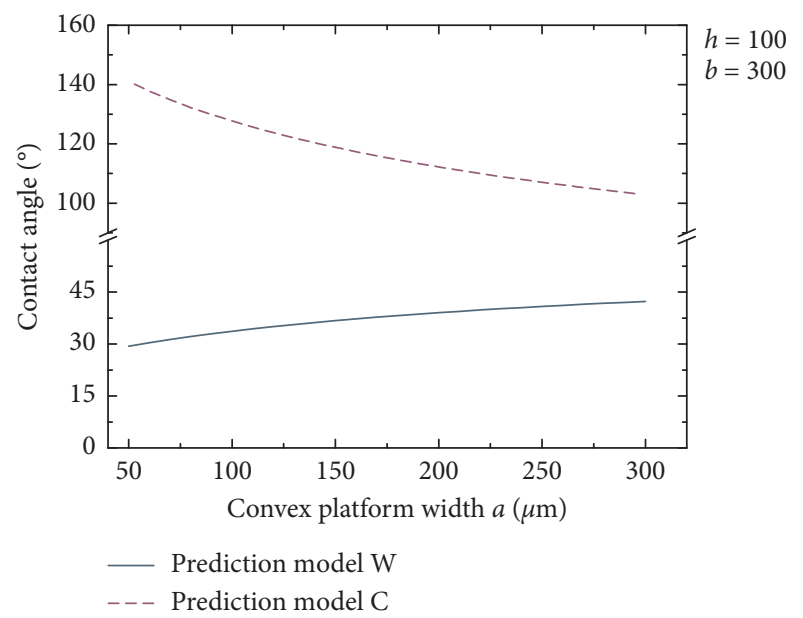

(a)

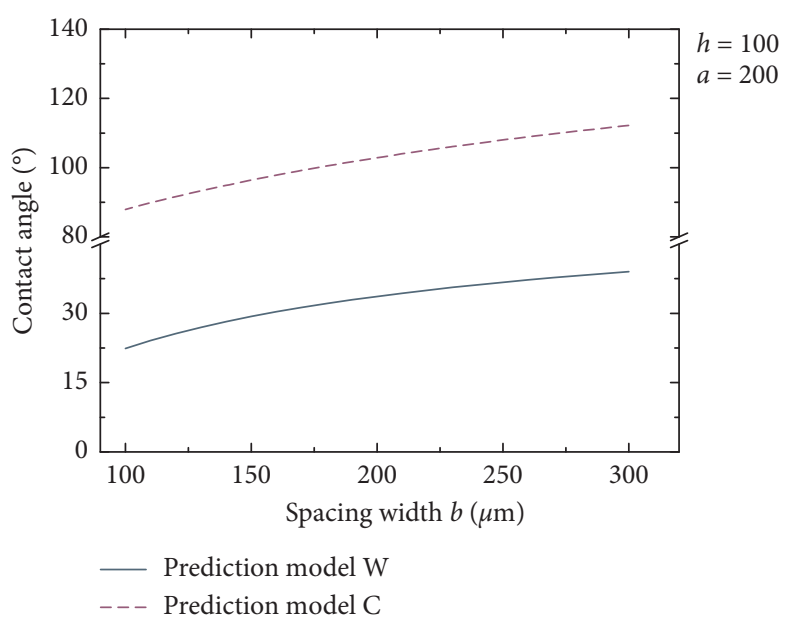

(b)

FIGURE 4: Law between structural parameters and contact angle: (a) convex platform width; (b) spacing width.

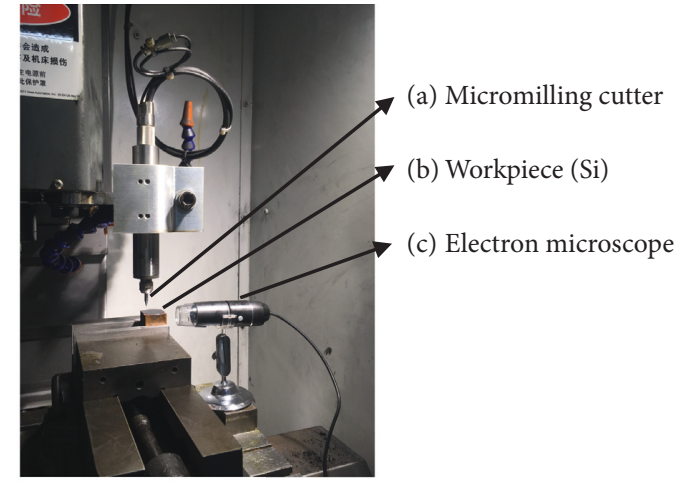

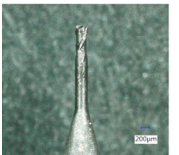

(a)

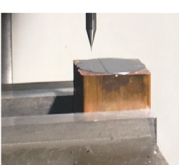

(b)

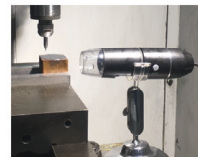

(c)
FIGURE 5: Schematic diagram of machining for the micromilling machine.

TABLE 1: Processing parameters of the grating structure.

\begin{tabular}{lccc}
\hline $\begin{array}{l}\text { Sample } \\
\text { number }\end{array}$ & $\begin{array}{c}\text { Height of } \\
\text { convex } h(\mu \mathrm{m})\end{array}$ & $\begin{array}{c}\text { Convex platform } \\
\text { width } a(\mu \mathrm{m})\end{array}$ & $\begin{array}{c}\text { Spacing width } \\
b(\mu \mathrm{m})\end{array}$ \\
\hline 1 & & 60 & \\
2 & & 90 & \\
3 & 100 & 120 & 300 \\
4 & & 150 & \\
5 & & 180 & \\
6 & & 200 & \\
\hline
\end{tabular}

Based on the analysis above, the contact angle of sample 1 was the largest and the hydrophobicity was the best. The further analysis on sample 1 was carried out.

Figure 9 shows the surface microstructure and size of sample 1, which was observed under 200 times of the VHX5000 ultradeep 3D microscope. Comparing Figures 9 and 10, it can see that there are some burrs on the edge of sample 1. As the tensile stress of monocrystalline silicon is much greater than its shear stress, curling is more easily to occur in the milling process of monocrystalline silicon. Then, the cracks and breakage will result from it. As a result, the experimental value of the contact angle is larger than the value of the theoretical model [42]. However, the overall gap is not so big, and the average $\mathrm{D}$ value is $4.3^{\circ}$. The overall contact angles achieve the desired results.

\section{Simulation Verification}

5.1. Model Establishment. Monocrystalline silicon is an octagonal diamond crystal structure with anisotropy. Combining with experimental, the 100 plane of single crystal silicon was selected for MD simulation. The grating micro-nanostructure model was constructed by removing the crystal cell of the model from the built model. Figure 11 is the simulation model in this experiment. The upper water molecules were arranged evenly, and the lower silicon took four atomic layer thicknesses.

In this simulation, the initial temperature of the system was $298 \mathrm{~K}$, and the integration step was $1.0 \mathrm{fs}$. The periodic boundary conditions were applied in the $X$ and $Y$ directions of the simulation space. Besides, the direction of $Z$ used the specular reflection boundary condition, and the simulation time was 800 ps [43]. The SPC model was used to simulate the droplets. Furthermore, the Lennard-Jones potential was used between the oxygen atoms, and the Tersoff potential was used to describe $\mathrm{Si}$ and $\mathrm{Si}$ in single crystal silicon. Lennard-Jones potential was used to describe the interaction between droplet and silicon substrate. Lennard-Jones potential energy was calculated as follows $[44,45]$ :

$$
u\left(r_{i j}\right)=4 \varepsilon\left[\left(\frac{\sigma}{r_{i j}}\right)^{12}-\left(\frac{\sigma}{r_{i j}}\right)^{6}\right],
$$

where $\varepsilon$ and $\sigma$ are energy parameters and length parameters, respectively. 


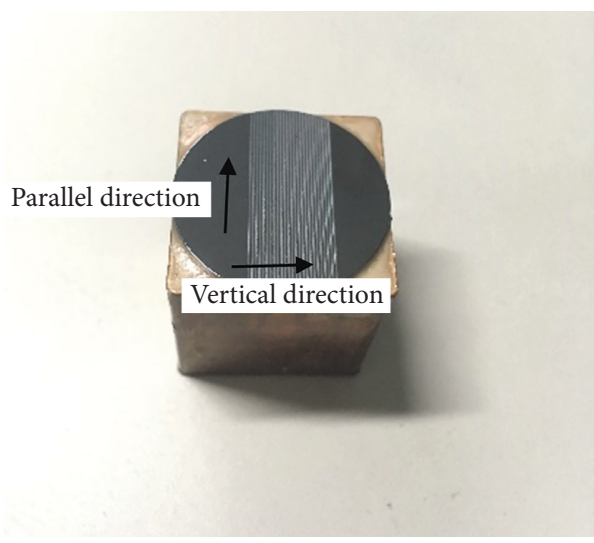

(a)

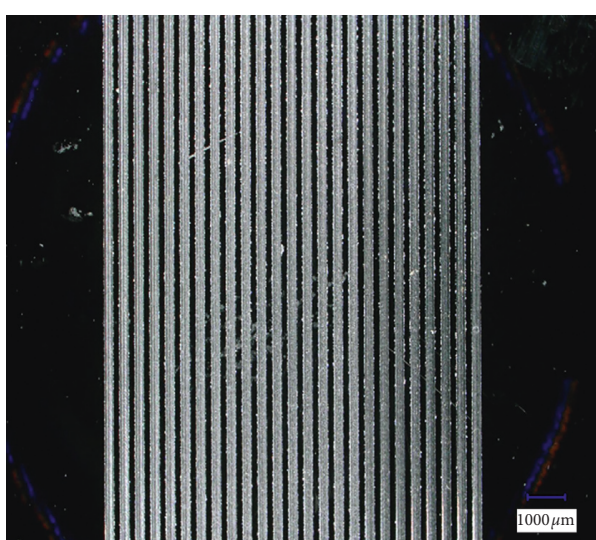

(b)

FIGURE 6: Surface diagram of the processed grating structure: (a) grating structure specimen; (b) grating structure micrograph.

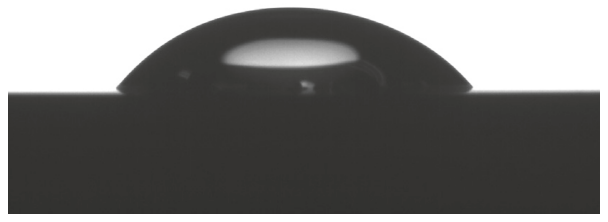

(a)

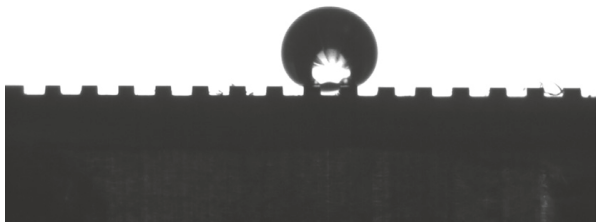

(b)

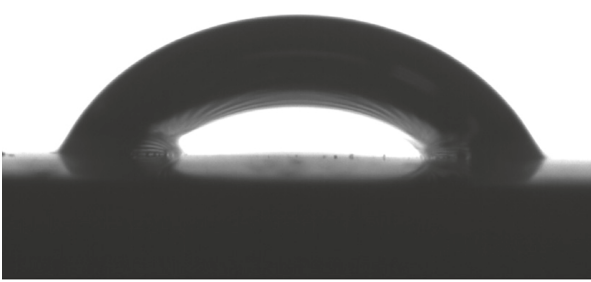

(c)

Figure 7: Contact angles of samples: (a) smooth surface; (b) parallel to the groove; (c) vertical to the groove.

TABLE 2: Theoretical and practical values of contact angle.

\begin{tabular}{lcccc}
\hline $\begin{array}{l}\text { Sample } \\
\text { number }\end{array}$ & $\begin{array}{c}\text { Vertical } \\
\text { to } \\
\text { the } \\
\text { groove } \\
\theta_{\mathrm{v}}\left({ }^{\circ}\right)\end{array}$ & $\begin{array}{c}\text { Parallel } \\
\text { to } \\
\text { the } \\
\text { groove } \\
\theta_{\mathrm{p}}\left({ }^{\circ}\right)\end{array}$ & $\begin{array}{c}\text { Prediction } \\
\text { model W } \\
\theta_{\mathrm{w}^{\prime}}\left({ }^{\circ}\right)\end{array}$ & $\begin{array}{c}\text { Prediction } \\
\text { model C } \\
\theta_{\mathrm{c}^{\prime}}\left({ }^{\circ}\right)\end{array}$ \\
\hline 1 & 82.8 & 138.7 & 30.8 & 136.4 \\
2 & 80.6 & 131.0 & 33.4 & 128.3 \\
3 & 74.2 & 128.4 & 35.6 & 122.1 \\
4 & 71.5 & 122.1 & 37.3 & 117.1 \\
5 & 67.4 & 119.9 & 38.8 & 112.9 \\
6 & 65.7 & 113.2 & 39.6 & 110.5 \\
\hline
\end{tabular}

The calculation formula of the Tersoff potential function for monocrystalline silicon was as follows:

$$
\begin{aligned}
u & =\sum u_{i}=\frac{1}{2} \sum_{i \neq j} u_{i j}, \\
u\left(r_{i j}\right) & =f_{\mathrm{C}}\left(r_{i j}\right)\left[f_{\mathrm{R}}\left(r_{i j}\right)+b_{i j} f_{\mathrm{A}}\left(r_{i j}\right)\right],
\end{aligned}
$$

where $u_{i j}$ is the potential function between atomic $i$ and atomic $j, f_{\mathrm{C}}\left(r_{i j}\right)$ is the truncated function, $f_{\mathrm{R}}\left(r_{i j}\right)$ is the

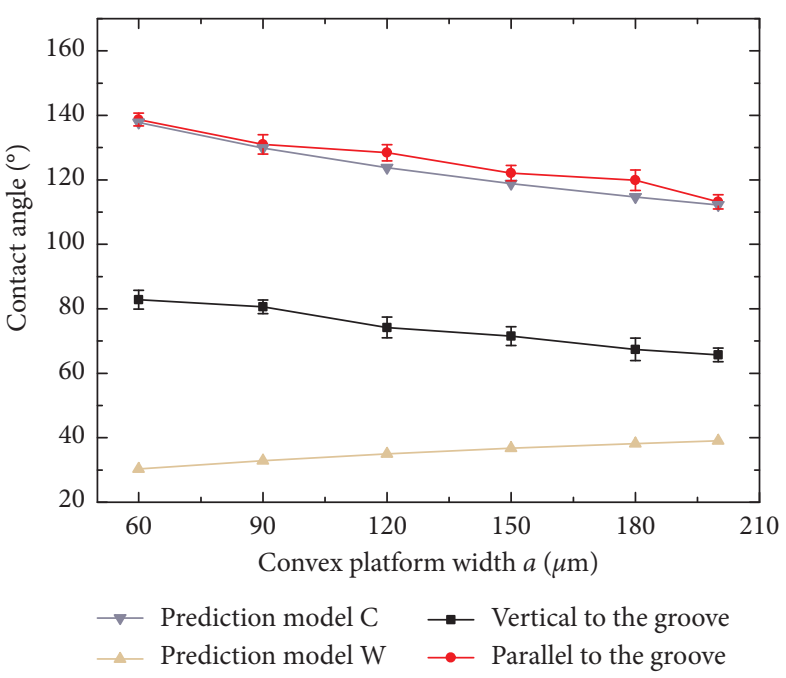

FIgURE 8: Variation curve of contact angle between measurement and theory.

repulsive function, $b_{i j}$ is the low order function, $f_{\mathrm{A}}\left(r_{i j}\right)$ is the attraction function, and $r_{i j}$ is the distance between atomic $i$ and atomic $j$. 

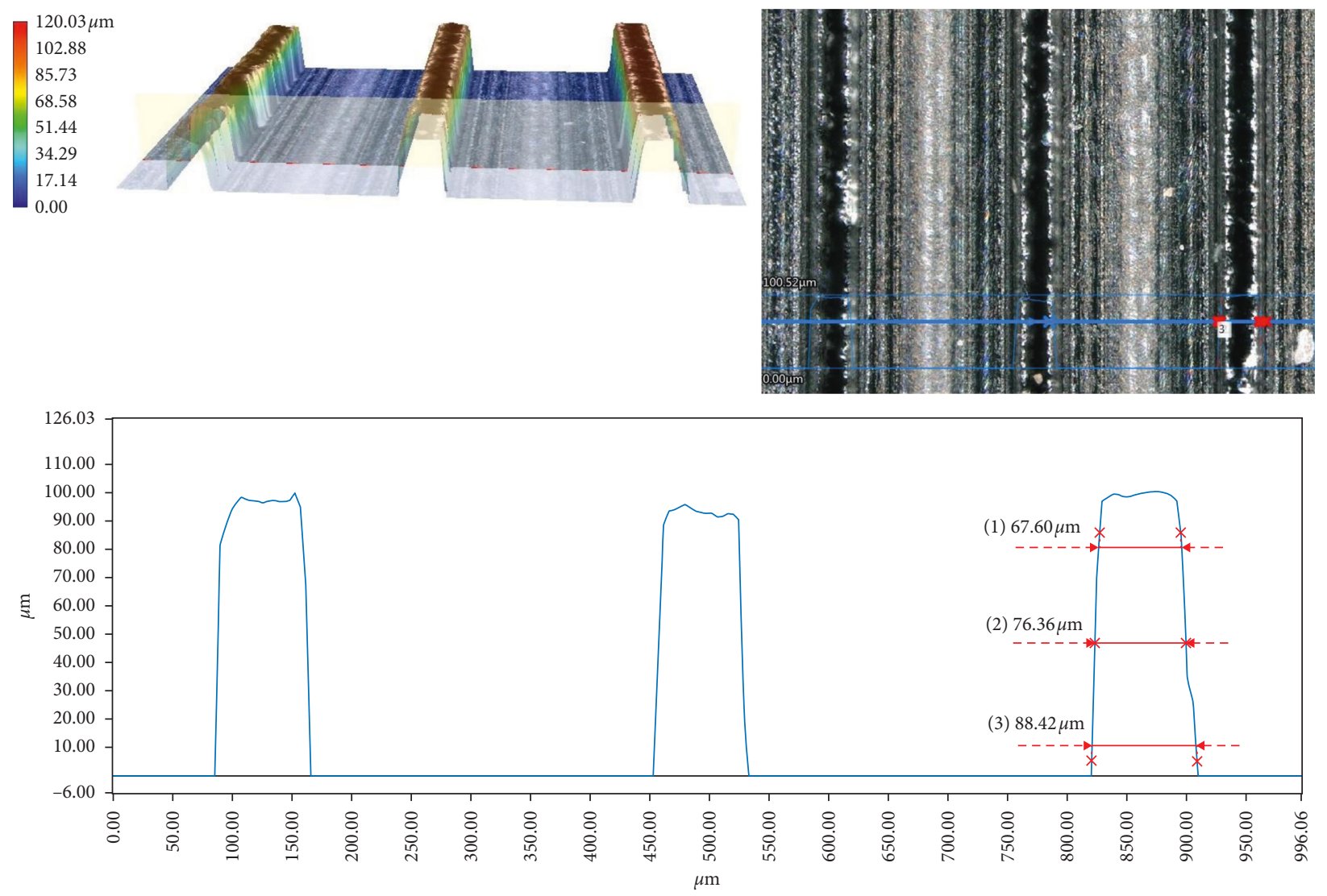

Figure 9: 3D topography of sample 1.

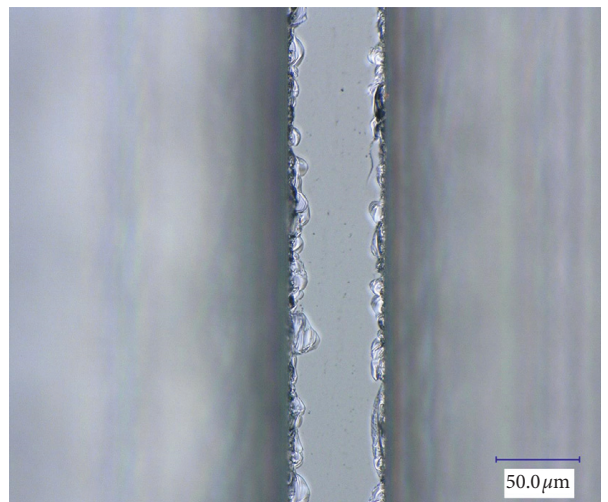

(a)

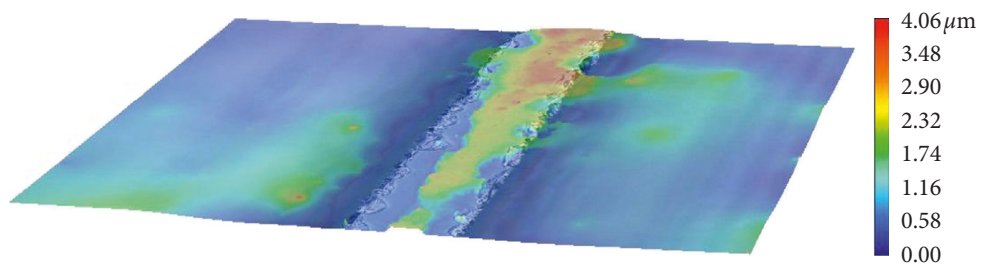

(b)

Figure 10: Microstructure topography of convex for sample 1: (a) microtopography of the convex surface at 1000 times; (b) threedimensional diagram of (a).

The potential energy parameters of the SPC model are shown in Table 3 [46].

5.2. Numerical Analysis. Figure 12(a) shows the droplet state under the surface of normal monocrystalline silicon. According to the previous simulation, it can be seen that the contact angle is $87^{\circ}$. Figure 12(b) shows the water droplet state under the grating structure. It is obvious that the contact angle of the grating structure is $124.4^{\circ}$. The hydrophobicity of the grating structure was verified by the molecular structure.

\section{Conclusion}

(1) A theoretical model of contact angle suitable for milling characteristics was established in this paper. The characteristics of the grating structure with micromilling were considered, and the accuracy of the theoretical model was guaranteed, which makes the experimental data more reliable.

(2) The contact angle in the parallel direction was always greater than that in the vertical direction. The droplets were mutually constrained in two 


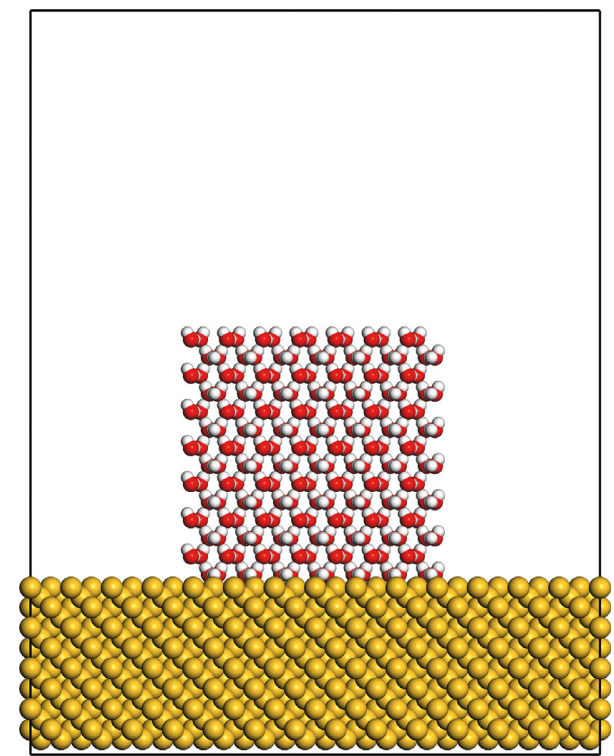

FIgURE 11: Molecular dynamics model without microstructure.

TABLE 3: Parameter values of the SPC model.

\begin{tabular}{lccccc}
\hline$r(\mathrm{OH})(\mathrm{nm})$ & $\theta\left(^{\circ}\right)$ & $q^{\mathrm{H}}(\mathrm{e})$ & $q^{\mathrm{O}}(\mathrm{e})$ & $\varepsilon_{0} k_{\mathrm{B}}^{-1}(\mathrm{~K})$ & $\sigma_{0}(\mathrm{~nm})$ \\
\hline 0.1 & 109.47 & 0.41 & -0.82 & 78.2 & 0.3166
\end{tabular}

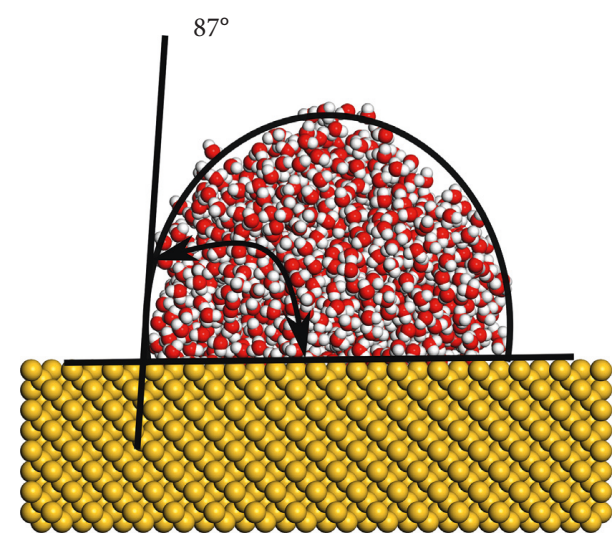

(a)

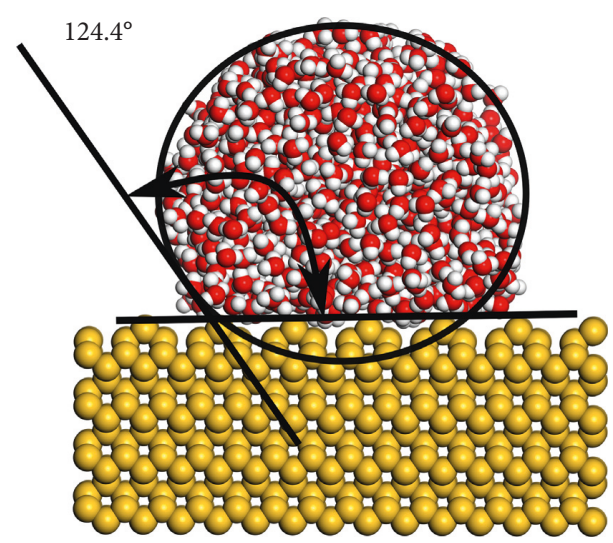

(b)

FIGURE 12: Simulation diagram of contact angle: (a) normal surface; (b) grating structure. directions, making the hydrophobic state more stable. The hydrophobic anisotropy of the grating structure can be beneficial to the special wetting conditions.

(3) The relationship between the contact angle and the size parameter of the grating structure was determined based on experimental data. For the grating structure with constant spacing width and convex height, the smaller the convex platform width, the larger the contact angle. The grating structure designed in this paper is simple, which can also ensure the improvement of the hydrophobicity of the material. There is good guidance for the construction of the hydrophobic surface on the grating structure.

(4) The difference between the experimental value and the theoretical value of the contact angle was due to the existence of some collapse edges on the edge of the convex. Overall, the contact angles of the grating structure achieved the desired results.

\section{Data Availability}

The data used to support the findings of this study were supplied by Ziyang Cao under license and so cannot be made freely available. Requests for access to these data should be made to the corresponding author upon request via mail.

\section{Conflicts of Interest}

The authors declare that they have no conflicts of interest.

\section{Acknowledgments}

The authors would like to acknowledge the support from the Research Fund Project of Suzhou University of Science and Technology (The study on surface integrity analysis and control technology of high-precision micromilling; XKZ201604), the National Natural Science Foundation of China (Research on the dynamics and stability in highprecision micromilling of microstructures; 51305286), and the international cooperation in high-level demonstration construction project of Jiangsu Province (Suzhou University of Science and Technology and the University of South Wales coorganized undergraduate education in mechanical design and manufacturing and automation; MOE32UK2A20121234N).

\section{References}

[1] N. J. Shirtcliffe, G. Mchale, S. Atherton, and M. I. Newton, "An introduction to superhydrophobicity," Advances in Colloid and Interface Science, vol. 161, no. 1-2, pp. 124-138, 2010.

[2] B. Widom, "Capillarity and wetting phenomena: drops, bubbles, pearls, waves," Physics Today, vol. 57, no. 12, p. 66, 2004.

[3] N. Gao, F. Geyer, D. W. Pilat et al., "How drops start sliding over solid surfaces," Nature Physics, vol. 14, no. 2, pp. 191-196, 2017. 
[4] N. J. Shirtcliffe, G. McHale, M. I. Newton, C. C. Perry, and P. Roach, "Superhydrophobic to superhydrophilic transitions of sol-gel films for temperature, alcohol or surfactant measurement," Materials Chemistry and Physics, vol. 103, no. 1, pp. 112-117, 2007.

[5] Y. Zhao, J. Li, J. Hu, L. Shu, and X. Shi, "Fabrication of superhydrophobic surfaces with long-term stability," Journal of Dispersion Science and Technology, vol. 32, no. 7, pp. 969-974, 2011.

[6] K. Yin, S. Yang, X. Dong, D. Chu, J.-A. Duan, and J. He, "Robust laser-structured asymmetrical PTFE mesh for underwater directional transportation and continuous collection of gas bubbles," Applied Physics Letters, vol. 112, no. 24, article 243701, 2018.

[7] Y. Wang and X. Gong, "Superhydrophobic coatings with periodic ring structured patterns for self-cleaning and oilwater separation," Advanced Materials Interfaces, vol. 4, no. 16, article 1700190, 2017.

[8] S. S. Latthe, R. S. Sutar, V. S. Kodag et al., "Self-cleaning superhydrophobic coatings: potential industrial applications," Progress in Organic Coatings, vol. 128, pp. 52-58, 2019.

[9] K. D. Kumar, I. A. Avramova, C. E. Castano et al., "Early stage anti-bioadhesion behavior of superhydrophobic soot based coatings towards Pseudomonas putida," Materials and Design, vol. 160, pp. 395-404, 2018.

[10] K. D. Mohammadi, C. E. Castano, R. Mohammadi et al., "Delayed condensation and frost formation on superhydrophobic carbon soot coatings by controlling the presence of hydrophilic active sites," Journal of Physics D: Applied Physics, vol. 51, no. 5, article 055302, 2018.

[11] K. Yin, H. Du, X. Dong, C. Wang, J.-A. Duan, and J. He, "A simple way to achieve bioinspired hybrid wettability surface with micro/nanopatterns for efficient fog collection," Nanoscale, vol. 9, no. 38, pp. 14620-14626, 2017.

[12] J. C. Zhang, J. Y. Liu, G. S. Wang, L. Huang, F. Z. Chen, and X. Liu, "Controllable wettability of laser treated aluminum mesh for on-demand oil/water separation," Journal of Dispersion Science and Technology, pp. 1-10, 2018.

[13] K. D. Esmeryan, C. E. Castano, A. H. Bressler, M. Abolghasemibizaki, and R. Mohammadi, "Rapid synthesis of inherently robust and stable superhydrophobic carbon soot coatings," Applied Surface Science, vol. 369, pp. 341-347, 2016.

[14] E.-Y. Mohammadi and J. Kim, "Effects of the boron-doped p+Emitter on the efficiency of the n-type silicon solar cell," Advances in Materials Science and Engineering, vol. 2013, Article ID 974507, 6 pages, 2013.

[15] S. M. R. Razavi, J. Oh, S. Sett et al., "Superhydrophobic surfaces made from naturally derived hydrophobic materials," ACS Sustainable Chemistry \& Engineering, vol. 5, no. 12, pp. 11362-11370, 2017.

[16] J. D. Wang, F. B. Liu, H. S. Chen, and D. Chen, "Superhydrophobic behavior achieved from hydrophilic surfaces," Applied Physics Letters, vol. 95, article 084104, 2009.

[17] Z. Chen, L. Hao, A. Chen, Q. Song, and C. Chen, "A rapid one-step process for fabrication of superhydrophobic surface by electrodeposition method," Electrochimica Acta, vol. 59, pp. 168-171, 2012.

[18] C. Liu, F. Su, J. Liang, and P. Huang, "Facile fabrication of superhydrophobic cerium coating with micro-nano flowerlike structure and excellent corrosion resistance," Surface and Coatings Technology, vol. 258, pp. 580-586, 2014.

[19] H. Yang, P. Pi, Z.-Q. Cai et al., "Facile preparation of superhydrophobic and super-oleophilic silica film on stainless steel mesh via sol-gel process," Applied Surface Science, vol. 256, no. 13, pp. 4095-4102, 2010.

[20] A. B. Gurav, S. S. Latthe, C. Kappenstein, S. K. Mukherjee, A. V. Rao, and R. S. Vhatkar, "Porous water repellent Silica coatings on glass by sol-gel method," Journal of Porous Materials, vol. 18, no. 3, pp. 361-367, 2011.

[21] V. Zorba, E. Stratakis, M. Barberoglou, E. Spanakis, P. Tzanetakis, and C. Fotakis, "Tailoring the wetting response of silicon surfaces via fs laser structuring," Applied Physics A: Materials Science \& Processing, vol. 93, pp. 819-825, 2008.

[22] O. Varlamova, K. Hoefner, M. Ratzke, J. Reif, and D. Sarker, "Modification of surface properties of solids by femtosecond LIPSS writing: comparative studies on silicon and stainless steel," Applied Physics A: Materials Science \& Processing, vol. 123, no. 12, p. 725, 2017.

[23] M. Conradi1, A. Drnovsek, and P. Gregorcic, "Wettability and friction control of a stainless steel surface by combining nanosecond laser texturing and adsorption of superhydrophobic nanosilica particles," Scientific Reports, vol. 8, no. 1, p. 7457, 2018.

[24] P. Gregorcic, M. Zupancic, and I. Golobic, "Scalable surface microstructuring by a fiber laser for controlled nucleate boiling performance of high- and low-surface-tension fluids," Scientific Reports, vol. 8, no. 1, p. 7461, 2018.

[25] Z. Y. Cao and H. Li, "Investigation of machining stability in micro milling considering the parameter uncertainty," Advances in Mechanical Engineering, vol. 7, no. 3, 2015.

[26] Z. Y. Cao, W. Y. Ding, and Z. Yin, "Research on surface integrity analysis and control technology of high precision milling," Journal of Advanced Oxidation Technologies, vol. 21, article 201801239, 2018.

[27] E. Bittoun and A. Marmur, "Optimizing super-hydrophobic surfaces: criteria for comparison of surface topographies," Journal of Adhesion Science and Technology, vol. 23, no. 3, pp. 401-411, 2009.

[28] M. Fathi, M. Abderrezek, and M. Friedrich, "Reducing dust effects on photovoltaic panels by hydrophobic coating," Clean Technologies and Environmental Policy, vol. 19, no. 2, pp. 577-585, 2017.

[29] C. Cui, X. L. Duan, B. Collier, and K. M. Poduska, "Fabrication and wettability analysis of hydrophobic stainless steel surfaces with microscale structures from nanosecond laser machining," Journal of Micro and Nano-Manufacturing, vol. 6, no. 3, article 031006, 2018.

[30] M. A. Rahman and A. M. Jacobi, "Wetting behavior and drainage of water droplets on microgrooved brass surfaces," Langmuir, vol. 28, no. 37, pp. 13441-13451, 2012.

[31] T. Young, "An essay on the cohesion of fluids," Philosophical Transactions of the Royal Society of London, vol. 95, pp. 65-87, 1805.

[32] C. Yang, F. He, and P. Hao, "The apparent contact angle of water droplet on the micro-structured hydrophobic surface," Science China Chemistry, vol. 53, no. 4, pp. 912-916, 2010.

[33] R. N. Wenzel, "Resistance of solid surfaces to wetting by water," Industrial \& Engineering Chemistry, vol. 28, no. 8, pp. 988-994, 1936.

[34] A. B. D. Cassie and S. Baxter, "Wettability of porous surfaces," Transactions of the Faraday Society, vol. 40, pp. 546-551, 1944.

[35] M. Nosonovsky and B. Bhushan, "Hierarchical roughness optimization for biomimetic superhydrophobic surfaces," Ultramicroscopy, vol. 107, no. 10-11, pp. 969-979, 2007.

[36] W. Sun, Dynamics and Heat Transfer of Condensed Droplets on Super-Hydrophobic Surfaces, Dalian University of Technology, Panjin, China, 2014. 
[37] X. Cheng, B. Gao, X. H. Yang, and J. Y. Liu, "Experimental study on the ductile-mode micro milling of single crystalline silicon," Journal of Shandong University of Technology (Natural Science Edition), vol. 26, pp. 53-55, 2012.

[38] Q. Gao, Y. D. Gong, M. Cai, and Y. G. Zhou, "Experimental study on surface quality in micro-milling of single crystal $\mathrm{Si}$ plastic removal," Technology and Test, vol. 6, pp. 135-138, 2016.

[39] A. Marmur, C. Della Volpe, S. Siboni, A. Amirfazli, and J. W. Drelich, "Contact angles and wettability: towards common and accurate terminology," Surface Innovations, vol. 5, no. 1, pp. 3-8, 2017.

[40] H. Song, "Micro-end milling and hydrophobic properties of machined surface for microgroove and microarray," Journal of Mechanical Engineering, vol. 52, no. 21, p. 198, 2016.

[41] S. Zhenyu, L. Zhanqiang, S. Hao, and Z. Xianzhi, "Prediction of contact angle for hydrophobic surface fabricated with micro-machining based on minimum Gibbs free energy," Applied Surface Science, vol. 364, pp. 597-603, 2016.

[42] Y. L. Wang, X. R. Zhang, L. X. Zhang, and Z. J. Yu, "Hydrophobic aluminum alloy surface fabricated by high speed micro-milling technology," China Surface Engineering, vol. 26, pp. 37-42, 2013.

[43] D. M. Huang, L. P. Wang, and Q. J. Xue, "Molecular dynamics simulation of AFM-based nanometric cutting of silicon," Tribology, vol. 31, pp. 328-334, 2011.

[44] C. S. Yuan, Investigation of Micro and Nano Structure on Titanium Alloy Surface Influences its Superhydrophobicity, Harbin Institute of Technology, Harbin, China, 2010.

[45] T. W. Kwon, J. Jang, M. S. Ambrosia, and M. Y. Ha, "Molecular dynamics study on the hydrophobicity of a surface patterned with hierarchical nanotextures," Colloids and Surfaces A: Physicochemical and Engineering Aspects, vol. 559, pp. 209-217, 2018.

[46] S. L. Yuan, H. Zhang, and D. J. Zhang, Molecular SimulationTheory and Experiment, Chemical Industry Press, Beijing, China, 2016. 


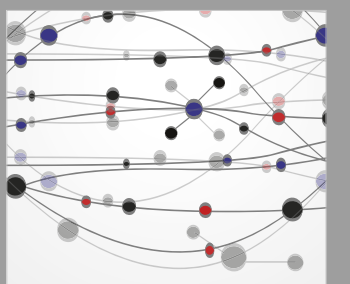

The Scientific World Journal
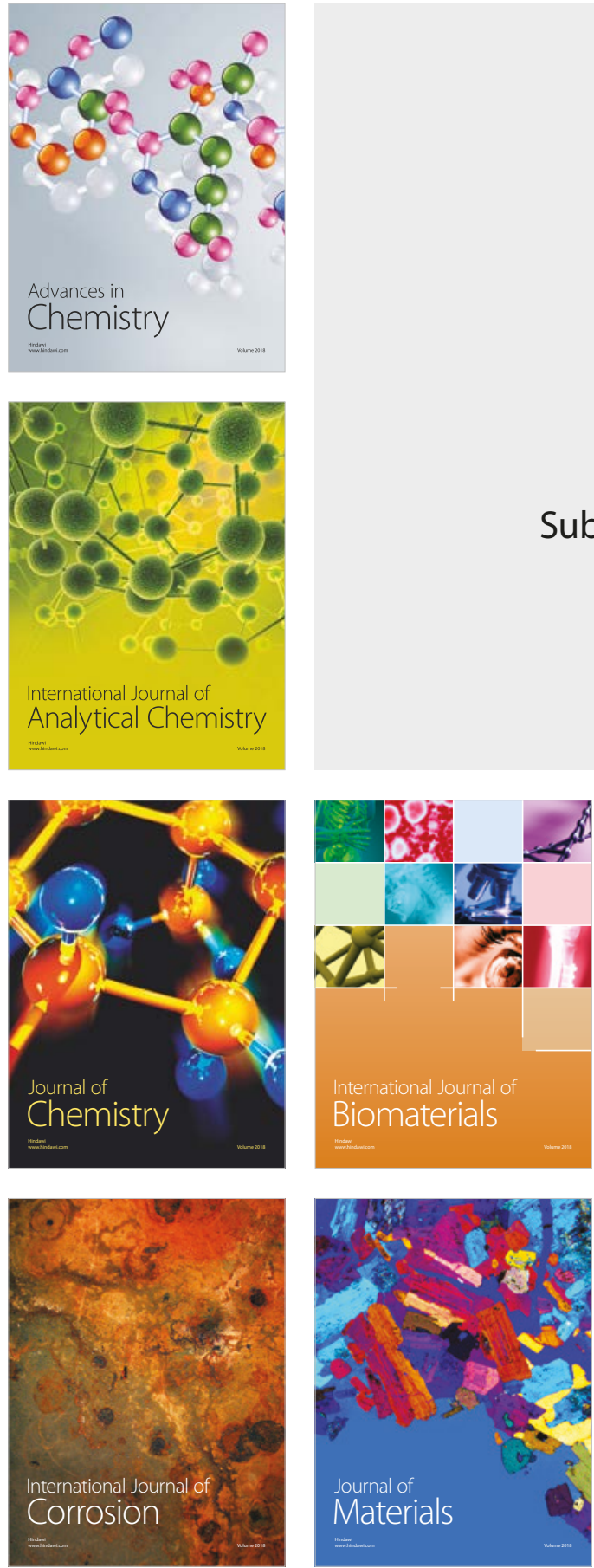

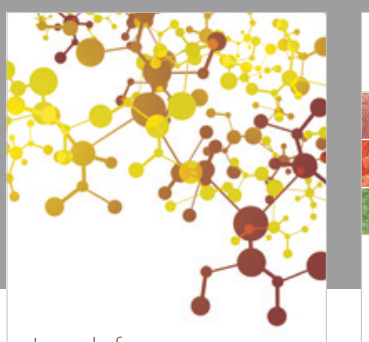

Journal of

Applied Chemistry
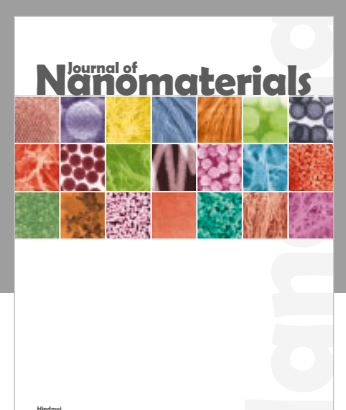

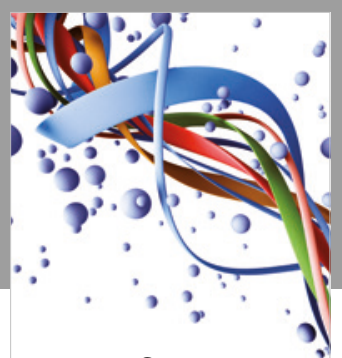

Scientifica

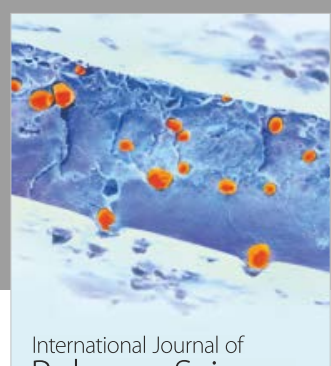

Polymer Science

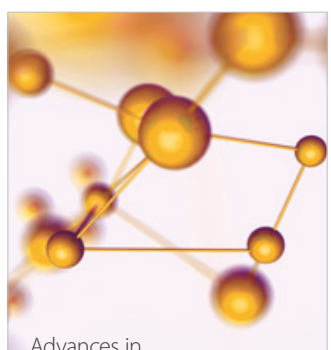

Physical Chemistry
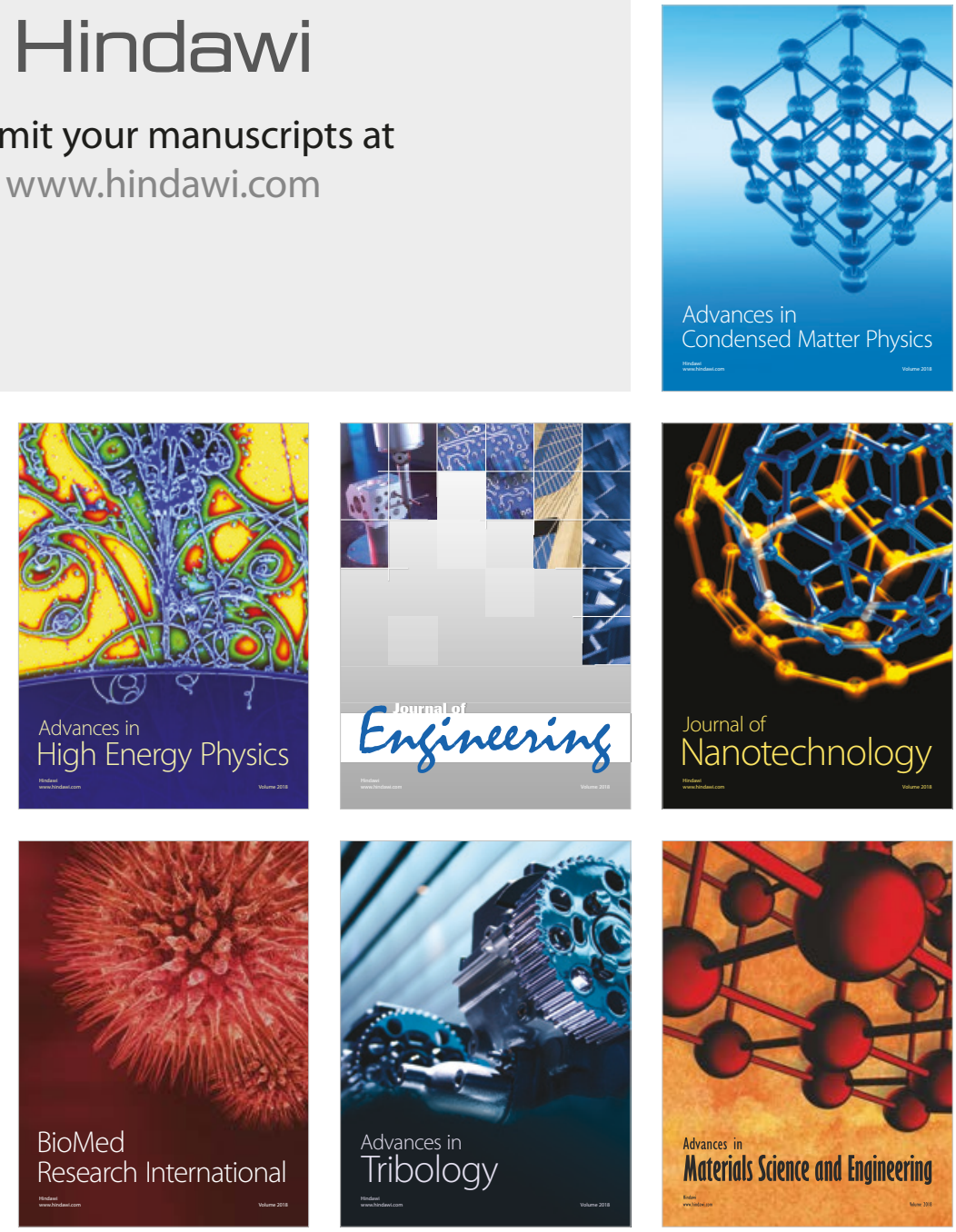